\title{
Why does Beijing's carbon emission of energy consumption have an 'inverted-W' shape during 2005-2015?
}

\author{
Zhihai Gong ${ }^{1,2,3}$, Junsong Jia ${ }^{1,2, *}$, Min Ju ${ }^{1,2}$ and Dongming Xie ${ }^{4}$ \\ ${ }^{1}$ Key Laboratory of Poyang Lake Wetland and Watershed Research, Ministry of Education, Jiangxi \\ Normal University, Nanchang, Jiangxi, 330022,China \\ ${ }^{2}$ School of Geography and Environment, Jiangxi Normal University, Nanchang, Jiangxi, 330022, \\ China \\ ${ }^{3}$ School of Graduate, Jiangxi Normal University, Nanchang, Jiangxi, 330022, China \\ ${ }^{4}$ Jiangxi Science \& Technology Normal University, Nanchang, Jiangxi, 330013, China \\ *jiaaniu@126.com
}

\begin{abstract}
Based on the characteristics of energy use and economic growth in Beijing, we analyzed the carbon emission of energy consumption from 2005-2015 by the Logarithmic Mean Divisia Index method (LMDI). The results show that Beijing's carbon emission of energy consumption had an 'inverted-W' shape during 2005-2015; the general trend of carbon emission was to rise first and then fall. The reasons were the follows. The economic scale effect was the leading factor for the increase of carbon emission in the first five years, with a contribution rate of $735.9 \%$. However, the energy intensity effect was the main driver of carbon emission suppression in the followed five years, with a contribution rate of $285.7 \%$. Therefore, some suggestions on the future control of carbon emission in Beijing's long-term development were put forward in the end.
\end{abstract}

Keywords: energy consumption; carbon emission; 'inverted-W' shape; LMDI; Beijing.

\section{Introduction}

With the rapid development of industrialization, oil and other fossil fuels' combustion created a large number of pollutants and greenhouse gases. Thus, as a result, the problem related to climate warming has become more and more prominent. The international community generally agreed that reducing greenhouse gas (especially $\mathrm{CO}_{2}$ emission) was the best way to solve the problem. Therefore, how to control and reduce the intensity of carbon emission is an important issue in the research of low-carbon economy in the nearest future. From different perspectives, many researchers analyzed the relationship between greenhouse gas emission reduction and economic development by the establishment of various theoretical and mathematical models. Sheinbaum [1], Ang [2] and Albrechtet [3], etc., measured the energy consumption of the United States, Japan and other countries, respectively. And their results are also used as an important monitor indicator to measure the energy efficiency, and as an important basis for making energy policy. Chinese academic such as Liu Y. and Teng F. [4], Wang H. T. [5] also researched on China's energy consumption and carbon emission from different perspectives.

\section{Data and methods}

We use the Logarithmic Mean Divisia Index method (LMDI) method and the model adopted in Ang B. W.'s [6] study to research the decomposition of Beijing energy consumption, basing on a large number of references. Due to limited space, the specific formula is ignored here. After decomposition, if the additive decomposition values are greater than zero, it means that the effect leads to an increase in $\mathrm{CO}_{2}$ emission, which in turn means that the effect leads to a reduction in $\mathrm{CO}_{2}$ emission. The data of $\mathrm{CO}_{2}$ emission, production value and energy consumption of the three industries are compiled according to Beijing Statistical Yearbook [7] from 2005 to 2015. In the calculation of energy consumption decomposition, we transform the consumption of various energy standards into the standard, referring to the National Development and Reform Commission announced conversion 
coefficient of various types of energy standard coal (coal, oil, natural gas, electricity conversion coefficients were $0.714,1.429,1.214,0.123$, respectively). The carbon emission coefficients of coal, oil and natural gas (Based on standard coal) are $0.748,0.583$ and 0.444 , respectively, according to data from the Energy Research Institute of the National Development and Reform Commission (NDRC).

\section{Empirical Analysis on Decomposition of Energy-consumption Carbon Emission in Beijing}

GDP and energy consumption in current situation. Beijing is one of the most poor areas whose energy resources are scare in China; its crude oil and natural gas are completely dependent on outsourcing, which cause its energy self-sufficiency rate very low, only about $2 \%$. In 2015, Beijing's GDP reached 2301.46 billion CNY which is an increase of about 7.3 times over 2000, with an average annual growth rate of $14.20 \%$. In the same time, the total energy consumption of Beijing is also increasing year by year likes the GDP. It shows that the growth of GDP had a driving effect on energy consumption. The total energy consumption in Beijing in 2015 was 68.526 million tons of standard coal, an increase of 1.7 times over 2000.

Change in energy consumption intensity. From 2000 to 2015, although energy consumption in Beijing was increasing, the intensity of energy consumption was declining, with an annual average decline of 9.4\%. From 2000 to 2008, Beijing's energy consumption intensity decreased significantly, with its energy efficiency interest rates continuing to increase, due to Beijing's industrial restructuring and the application and promotion of high-tech. But from 2008 on, the trend of decline in Beijing's energy consumption intensity tended to ease. (see Fig.1)

Decomposition results and analyses. Beijing's energy-consumption carbon emission increased from 2005 to 2007, but which began to decline from 2007 to 2008. While it increased again from 2008-2010, and it decreased again from 2010-2011. At last, it decreased from 2012-2015 continuously after in a short increase from 2011-2012. The shape likes an 'inverted W'. (see Fig.1)
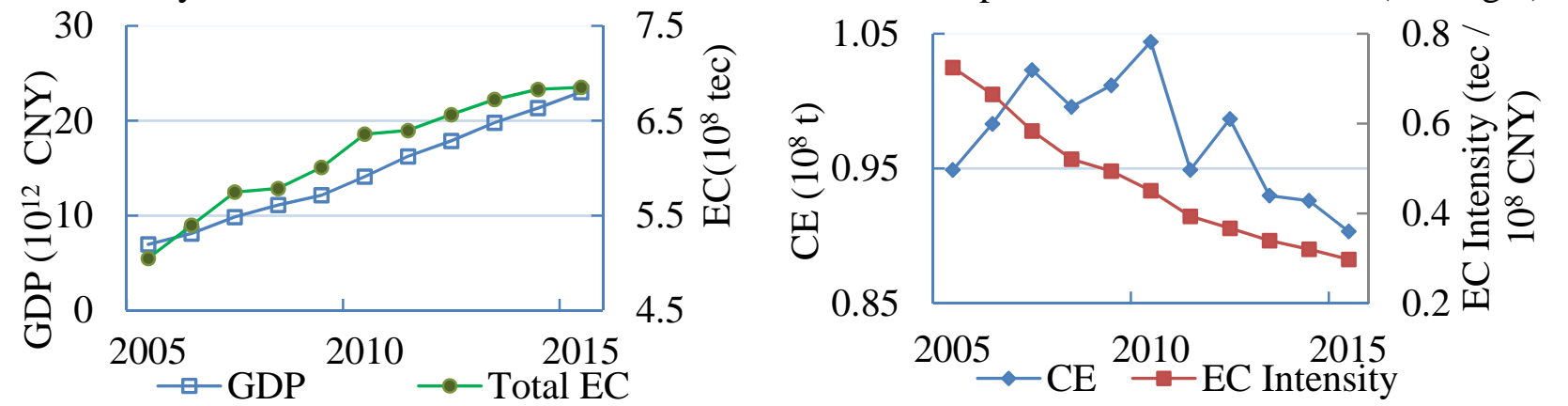

Figure.1 GDP, Energy Consumption (EC) and Carbon Emission (CE), EC Intensity of Beijing from 2000-2015

From the decomposition effect showed in Fig.2, the energy intensity effect greatly reduced the carbon emission, so the energy intensity effect was the slowing factor of carbon emission growth. But the economic scale effect was positively related to the growth of carbon emission, so economic growth was a contributing factor to the growth of carbon emission. The details are analyzed in follows.

First of all, the economic scale effect had a positive impact on each industry's carbon emission from 2005-2015. And the contribution of carbon emission on total economic scale effect was particularly larger than any other effects. What's more, the trend of this contribution is consistent with the trend of carbon emission in Beijing, indicating that the economic scale effect was an important source of the 'inverted W' shape on carbon emission. In the next place, the industrial structure effect had a main positive impact of carbon emission suppression in the first and second industry with its trend also being consistent with that in carbon emission, indicating that the 'inverted W' shape of Beijing's carbon emission is closely related to the industrial restructuring in primary industry and secondary industry. As for the energy intensity effect, the total contribution rates of $\mathrm{CO}_{2}$ emission suppression in the primary industry, secondary industry and tertiary industry were $10.60 \%, 1051.1 \%$ and 665.8\%, respectively (see Table.1), indicating that the decrease of carbon emission was mainly 
due to the improvement of energy efficiency of the secondary industry, followed by the tertiary industry. At the same time, the trend of the contribution on carbon emission suppression is also consistent with that on carbon emission, indicating that the energy intensity effect was another important factor leading to the 'inverted W' shape. Last but not least, the industrial emission coefficient effect actually reflects the energy structure of industry. In recent years, Beijing vigorously eliminated the backward production capacity, and enhanced the development of energy-saving industries, thus the proportion of clean energy use was greatly improved. In the industrial emission coefficient effect, the total contribution rate of the first, second and third industries were 8.71\%, $305.75 \%$ and $158.4 \%$, respectively (see Table.1), which means that the role of energy structure optimization to reduce carbon emission also cannot be ignored. Although this result exists, its affection on the 'inverted $W$ ' shape was not so distinguished, because its trend is not entirely consistent with this shape.

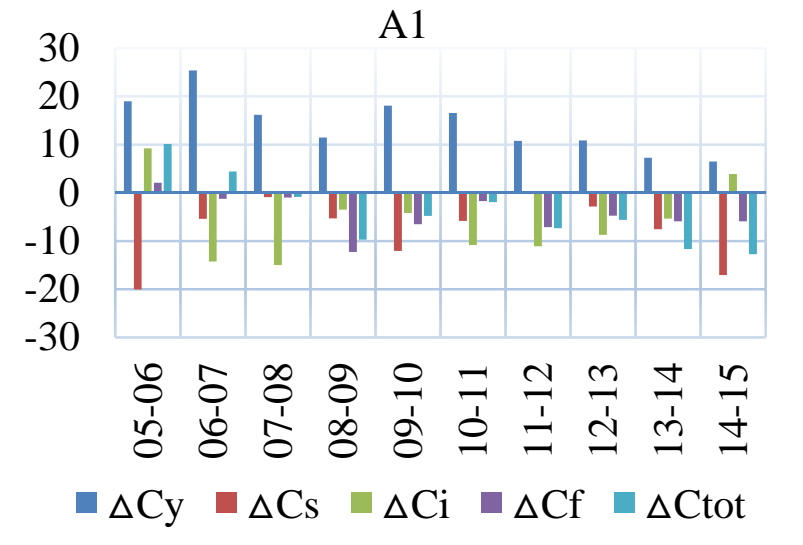

A3

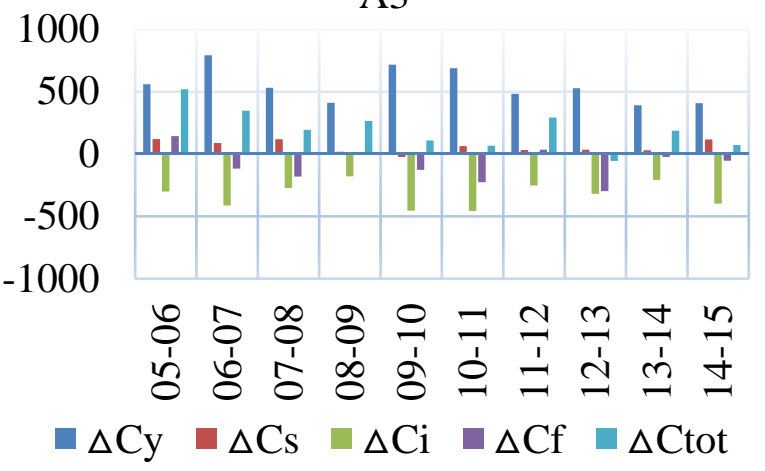

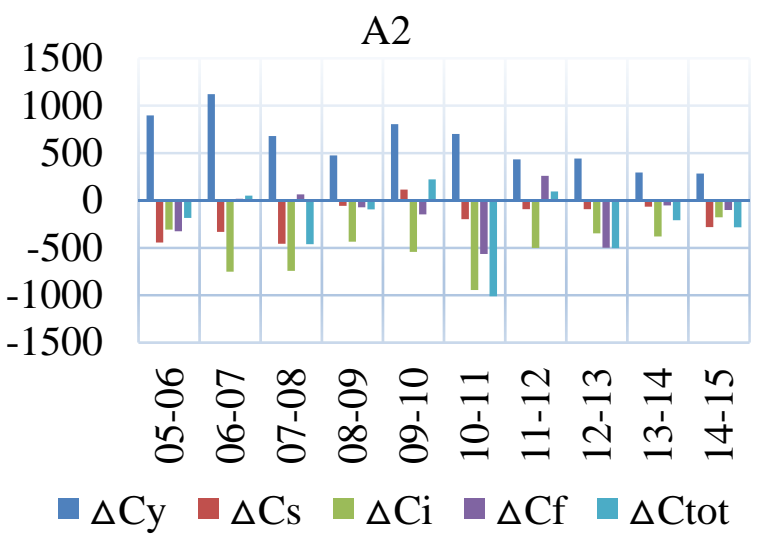

Total

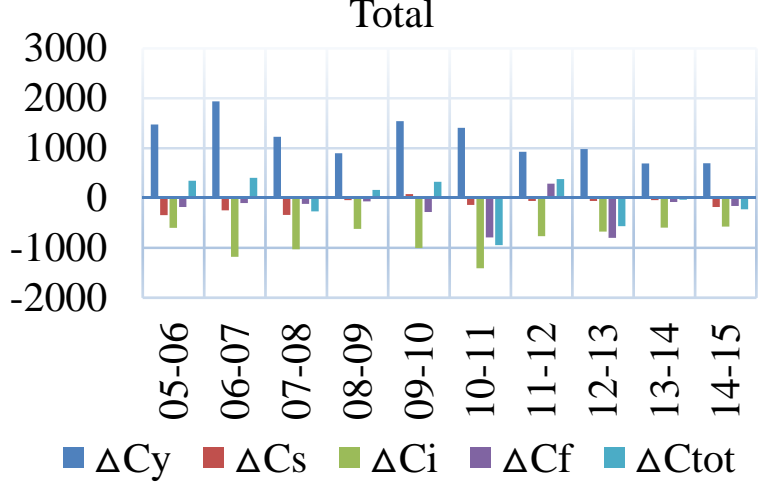

Figure.2 Four Effects of Different Industries in Beijing from 2006 to 2015. (A1, A2, A3 represent the

first, second and tertiary industries respectively, $\Delta \mathrm{C}_{\mathrm{y}}, \Delta \mathrm{Cs}, \Delta \mathrm{Ci}, \Delta \mathrm{Cf}$ and $\Delta \mathrm{C}_{\text {tot }}$ represent economic scale effect, industrial structure effect, energy intensity effect, energy-consumption carbon emission and total effect, respectively, 05-06 refers to year 2005-2006. Unit: $10^{4}$ tonnes (t). )

In total, the economic scale effect, industrial structure effect and energy intensity effect was the main reason in why Beijing's carbon emission of energy consumption had an 'inverted-W' shape during 2005-2015. In addition, if we phase this curve into two periods (2005-2010, and 2010-2015), we can find that the carbon emission increased from 2005-2010 while decreased from 2010-2015 in general. As shown in Table1, the total contribution rates of four effects on carbon emission suppression were $735.9 \%,-94.01 \%,-466.4 \%$ and $-75.53 \%$, respectively, indicating that the economic scale effect was the main factor of carbon emission during 2005-2010, instead of energy intensity effect, although it decreased the most carbon emission to a certain degree. That is why Beijing's carbon emission increased from 2005 to 2010. As for 2010-2015, the total contribution rates of four effects on carbon emission suppression were $-335.8 \%, 39.34 \%, 285.7 \%, 110.83 \%$, respectively, accounting for the energy intensity effect as the main factor of carbon emission suppression during 2010-2015. Therefore, because the energy intensity can reduce most carbon emission, Beijing's carbon emission decreased from 2010 to 2015. 
Table.1 Factorization contribution rate of Beijing's carbon emission from energy-consumption during 2005-2015(\%)

\begin{tabular}{|c|c|c|c|c|c|c|c|c|c|c|c|}
\hline \multirow{2}{*}{$\begin{array}{c}\text { Decompositi } \\
\text { on Item }\end{array}$} & \multicolumn{4}{|c|}{$2005-2010$} & \multicolumn{4}{|c|}{ 2010-2015 } & \multicolumn{3}{|c|}{ 2005-2015 } \\
\hline & $\mathrm{A} 1$ & $\mathrm{~A} 2$ & A3 & Total & A1 & $\mathrm{A} 2$ & A3 & Total A1 & $\mathrm{A} 2$ & A3 & Total \\
\hline$\triangle \mathrm{Cy}$ & 8.80 & 424.1 & 0303.07 & 735.9 & -3.37 & -154.6 & -177.8 & $-335.8-25.54$ & +-122 & -1135. & -2381. \\
\hline$\triangle \mathrm{Cs}$ & -4.39 & -123. & 533.97 & -94.01 & 2.45 & 56.04 & -19.15 & 39.3415 .14 & 391.1 & $1-125$ & 281.0 \\
\hline$\triangle \mathrm{Ci}$ & -2.62 & -300 & $4-163.3$ & -466.4 & 1.97 & 167.3 & 116.4 & 285.7010 .60 & 1051 & 1665.8 & 1727. \\
\hline$\triangle \mathrm{Cf}$ & -1.89 & -50.3 & $5-23.28$ & -75.53 & 1.76 & 68.16 & 40.91 & 110.838 .71 & 305.7 & 3.4 & 472.9 \\
\hline$\triangle$ Ctot & -0.11 & -50.2 & 5150.36 & 100.0 & 2.81 & 136.8 & -39.64 & 100.008 .91 & 527. & $2-450.0$ & 100.0 \\
\hline
\end{tabular}

\section{Conclusions}

According to the comprehensive analysis of energy consumption and economic growth trends, we can also reveal a deeper reason that Beijing's economic development has entered a high level stage, with the initial realization of the carbon emission reduction through the development of energy-saving industries and restrictions in the use of private cars. The corresponding energy and resource consumption is tending to increase, while the carbon emission base is still large, although the low-carbon industry share rose rapidly. In view of this, Beijing should continue to phase out the development model of high energy-consuming industrial economy, which is as the leading economic, and transform to the economic development model of diversified, environmental protection, energy saving, high value-added. Only if taking the development of low-carbon economy, and gradually get rid of heavy chemical industry economy, at the same time improve the energy structure and new energy varieties, can Beijing's economy achieve the sustainable development.

\section{Acknowledgements}

We are grateful for the financial support provided by Chinese National Science Foundation (71473113, 31360120, 51408584) and Natural Science Foundation of Jiangxi (20151BAB203040). Corresponding author Junsong Jia can be contacted by (86)18607918843 or jiaaniu@126.com.

\section{References}

[1] C. Sheinbaum, L. Ozawa, D. Castillo. Using logarithmic mean Divisia index to analyze changes in energy use and carbon dioxide emissions in Mexico's iron and steel industry. Energy Economics, 2010, 32(6):1337-1344.

[2] B. W. Ang, F. R. Liu. A new energy decomposition method: perfect in decomposition and consistent in aggregation. Energy, 2001, 26(6):537-548.

[3] J. Albrecht, D. Francois, K. A. Schoors, A shapley decomposition of carbon emission without residuals. Energy Policy, 2002, 30(9):727-736.

[4] Y. Liu, F. Teng, J. Song, C. Baumont. Decomposition Analysis of Changes in Metropolitan Energy Consumption in China Based on the Logarithmic Mean Divisia Index. Journal of Resources and Ecology, 2012, 5(3):228-236.

[5] H. T. Wang. Logarithmic mean divisia index model and the carbon emission mechanism of energy sector in Shanghai. China Population Resources \& Environment, 2010, 20(5):143-146.

[6] B. W. Ang. The LMDI approach to decomposition analysis: a practical guide. Energy Policy, 2005, 33(7):867-871.

[7] Information on http://www.bjstats.gov.cn. 